\title{
Adsorption of charged and neutral polymer chains on silica surfaces: The role of electrostatics, volume exclusion, and hydrogen bonding
}

\author{
Evan Spruijt,,$^{1,2, *}$ P. M. Biesheuvel,,${ }^{3,4, \dagger}$ and Wiebe M. de Vos ${ }^{5, \dagger}$ \\ ${ }^{1}$ ESPCI ParisTech, Physique et Mécanique des Milieux Hétérogènes, UMR 7636 du CNRS, 10 rue Vauquelin, 75005 Paris, France \\ ${ }^{2}$ Radboud University Nijmegen, Institute for Molecules and Materials, Heyendaalseweg 135, 6525 AJ Nijmegen, The Netherlands \\ ${ }^{3}$ Wetsus, European Centre of Excellence for Sustainable Water Technology, Oostergoweg 9, 8911 MA Leeuwarden, The Netherlands \\ ${ }^{4}$ Wageningen University, Laboratory of Physical Chemistry and Colloid Science, Dreijenplein 6, 6703 HB The Netherlands \\ ${ }^{5}$ University of Twente, Membrane Science and Technology, MESA ${ }^{+}$Institute of Nanotechnology, P.O. Box 217, 7500 AE Enschede,
}

The Netherlands

(Received 7 October 2014; published 12 January 2015)

\begin{abstract}
We develop an off-lattice (continuum) model to describe the adsorption of neutral polymer chains and polyelectrolytes to surfaces. Our continuum description allows taking excluded volume interactions between polymer chains and ions directly into account. To implement those interactions, we use a modified hard-sphere equation of state, adapted for mixtures of connected beads. Our model is applicable to neutral, charged, and ionizable surfaces and polymer chains alike and accounts for polarizability effects of the adsorbed layer and chemical interactions between polymer chains and the surface. We compare our model predictions to data of a classical system for polymer adsorption: neutral poly( $N$-vinylpyrrolidone) (PVP) on silica surfaces. The model shows that PVP adsorption on silica is driven by surface hydrogen bonding with an effective maximum binding energy of about $1.3 k_{\mathrm{B}} T$ per PVP segment at low $p \mathrm{H}$. As the $p \mathrm{H}$ increases, the $\mathrm{Si}-\mathrm{OH}$ groups become increasingly dissociated, leading to a lower capacity for $\mathrm{H}$ bonding and simultaneous counterion accumulation and volume exclusion close to the surface. Together these effects result in a characteristic adsorption isotherm, with the adsorbed amount dropping sharply at a critical $p \mathrm{H}$. Using this model for adsorption data on silica surfaces cleaned by either a piranha solution or an $\mathrm{O}_{2}$ plasma, we find that the former have a significantly higher density of silanol groups.
\end{abstract}

DOI: 10.1103/PhysRevE.91.012601

\section{INTRODUCTION}

The adsorption of polymer chains onto surfaces has been investigated extensively since the discovery in the 1950s that particle suspensions could be stabilized against flocculation by layers of adsorbed polymer chains [1-3]. Stabilization of particle suspensions poses a critical step in the development of many products used in everyday life, such as paints, detergents, cosmetics, and pesticides [1-6]. Particle stabilization is equally important for many novel applications related to drug delivery and particle-based analysis of complex media [7-10]. In all these cases, a detailed understanding of the physicochemical nature of polymer adsorption is indispensable.

Many features of polymer adsorption are now well understood. For polymer adsorption to take place, the adsorption energy per segment must be higher than a critical value [3]. Upon adsorption polymer chains lose both conformational and translational freedom, which must be compensated for by the adsorption energy. For long polymer chains the loss of conformational freedom will dominate the total entropy loss. An adsorbed polymer chain is commonly described as a series of trains, loops, and tails [3,11,12]. De Gennes [11,12], translated this simple qualitative description of polymer adsorption into clear quantitative predictions of the density profile of adsorbed polymer chains. Three regions of the density profile can be distinguished: (i) the proximal region

\footnotetext{
*e.spruijt@ science.ru.nl

†maarten.biesheuvel@wur.nl

${ }^{\ddagger}$ w.m.devos@utwente.nl
}

PACS number(s): 82.35.Lr, 68.47.Pe, 64.10.+h, 82.30.Rs

close to the surface has the highest concentration of polymer chains (trains) and is affected most directly by the exact nature of the polymer-surface interactions, (ii) the central region is made up of loops and tails with a density that decreases following a power law, and (iii) the distal region is dominated by tails, with an exponentially decreasing density.

Two of the most widely used experimental model systems to study the physics of polymer adsorption consist of nonionic polymers adsorbing onto negatively charged silica surfaces in water. Both poly(ethylene oxide) (PEO) and $\operatorname{poly}(N$ vinylpyrollidone) (PVP) have received much attention [1320]. Experiments have been carried out on both colloidal silica and planar silica surfaces, using NMR, ellipsometry, reflectometry, and x-ray and neutron scattering and reflection. Neutron reflection and neutron scattering measurements showed typical density profiles to be mostly exponential [20], in line with De Gennes' predictions for the distal region, while NMR enabled differentiation between trains on the one hand and loops and tails on the other [16]. Finally, the adsorption energy of PVP [14] and PEO [21] to silica was determined experimentally by measuring the desorption transition in binary solvents.

The chemical nature of most surfaces to which polymer chains adsorb, including the model silica and mica surfaces, depends strongly on the solution properties. The surface of silica consists of siloxane ( $\mathrm{Si}-\mathrm{O}-\mathrm{Si})$ and silanol $(\mathrm{Si}-\mathrm{OH})$ groups [22]. The degree of dissociation of the silanol groups (to $\left.\mathrm{Si}^{-} \mathrm{O}^{-}\right)$, and thus the surface charge, varies dramatically with the $p \mathrm{H}$ and ionic strength of the aqueous solution [23]. Van der Beek et al. showed that, as a consequence, the adsorption to silica of the two model polymers, PVP and PEO, depends 
on the $p \mathrm{H}$ as well, with the adsorbed amount dropping sharply between $p \mathrm{H} 9$ and 11 [16]. Others showed that an increase in ionic strength leads to a decrease in the adsorbed amount for both PEO [24] and PVP [25]. Hydrogen bonding with $\mathrm{Si}-\mathrm{OH}$ groups is the driving force for the adsorption of both PEO and PVP to silica $[13,16]$. An increase in either $p \mathrm{H}$ or ionic strength, or both, leads to dissociation of silanol groups and therefore to a decreased hydrogen bonding capacity. In addition, the concentration of counterions close to the surface increases dramatically at high surface charge, leading to significant excluded volume interactions between ions and polymer chains and further polymer chain desorption.

Recently, De Vos et al. showed that the adsorption of PVP to a silica surface also depends on the method used to clean and prepare the silica surface [25]. For an $\mathrm{O}_{2}$ plasma-cleaned silica surface, a drop in polymer adsorption was observed between $p \mathrm{H} 7$ and 10, while for piranha-cleaned silica surface the drop was observed between $p \mathrm{H} 9$ and 11 . The difference was attributed to different silanol densities resulting from the surface treatments.

Based on the existing experiments at a wide range of $p \mathrm{H}$ and salt concentrations, it is clear that the ionizable character of silica plays a key role in the adsorption of neutral polymer chains such as PVP and PEO. Nevertheless, only few theoretical models describe and predict their adsorption as a function of $p \mathrm{H}$ and ionic strength. Pattanayek and Juvekar developed a model for the adsorption of PEO to silica at different $p \mathrm{H}$ with a $p \mathrm{H}$-dependent attraction parameter [26]. Their model did not include ionic strength or excluded volume effects. Postmus et al. used lattice-based, numerical self-consistent field theory to take the volume of ions explicitly into account [24]. In their model the adsorption energy was a function of the density of $\mathrm{Si}-\mathrm{OH}$ groups and they found qualitative agreement with experimental data. A full quantitative comparison proved difficult due to the lattice-based approach in which Kuhn length, monomer size, and ion size are all coupled parameters.

In this paper we give an off-lattice (continuum) description of polymer adsorption to a charged, hard surface, making use of the Edwards equation in the ground-state dominance approximation, combined with a modified Poisson-Boltzmann equation for the (polymer-modified) electrical double layer that develops next to the silica surface. Both ionizable surfaces (silica) and polymer chains can be included in a self-consistent way in the electrostatic calculation. Excluded volume effects of both polymer chains and ions are taken into account using a recently developed off-lattice model, modified to account polymer connectivity. Here we apply this equation of state for the first time to the case of polymer adsorption. Our model based on hydrogen bonding between $\mathrm{Si}-\mathrm{OH}$ surface groups and the polymer chains correctly predicts the experimentally measured $p \mathrm{H}$-dependent adsorption-desorption isotherm of a neutral model polymer, poly(vinyl pyrrolidone) (PVP) to silica [25]. The adsorbed amount of PVP decays sharply for $p \mathrm{H} \gg p K_{\mathrm{a}, \mathrm{s}}$, in good agreement with experimental data, due to the combined deprotonation of $\mathrm{Si}-\mathrm{OH}$ groups and excluded volume interactions between polymer chains and ions that accumulate close to the charging silica surface. By contrast, alternative models in which the surface-polymer interactions are independent of the $\mathrm{SiOH}$ density or based on attractive electrostatic interactions cannot describe the experimental data.

\section{THEORY}

We consider a solution of polymer chains and ions (see Fig. 1). In general, a polymer chain of contour length $L=a N$ can be divided in various ways in $N$ segments of length $a$. One possible choice for the segment length, which we adopt in this paper, is the Kuhn length, capturing the flexibility of the polymer chains. The polymer chains can be either neutral or charged. When comparing our model with experimental data in Sec. III we will consider only neutral polymer chains of poly(vinylpyrrolidone) (PVP), but here we first give a general description that also applies to polyelectrolytes.

We define a polymer line charge density, i.e., the charge per unit length along the polymer backbone, $\alpha_{\mathrm{p}} \lambda$, where $\alpha_{\mathrm{p}}$ is the ionization degree $\left(\alpha_{\mathrm{p}}=1\right.$ for strong or quenched polyelectrolytes, and $0<\alpha_{\mathrm{p}}<1$ for weak or ionizable polyelectrolytes). The maximum line charge density $\lambda$ (unit $\mathrm{m}^{-1}$ )

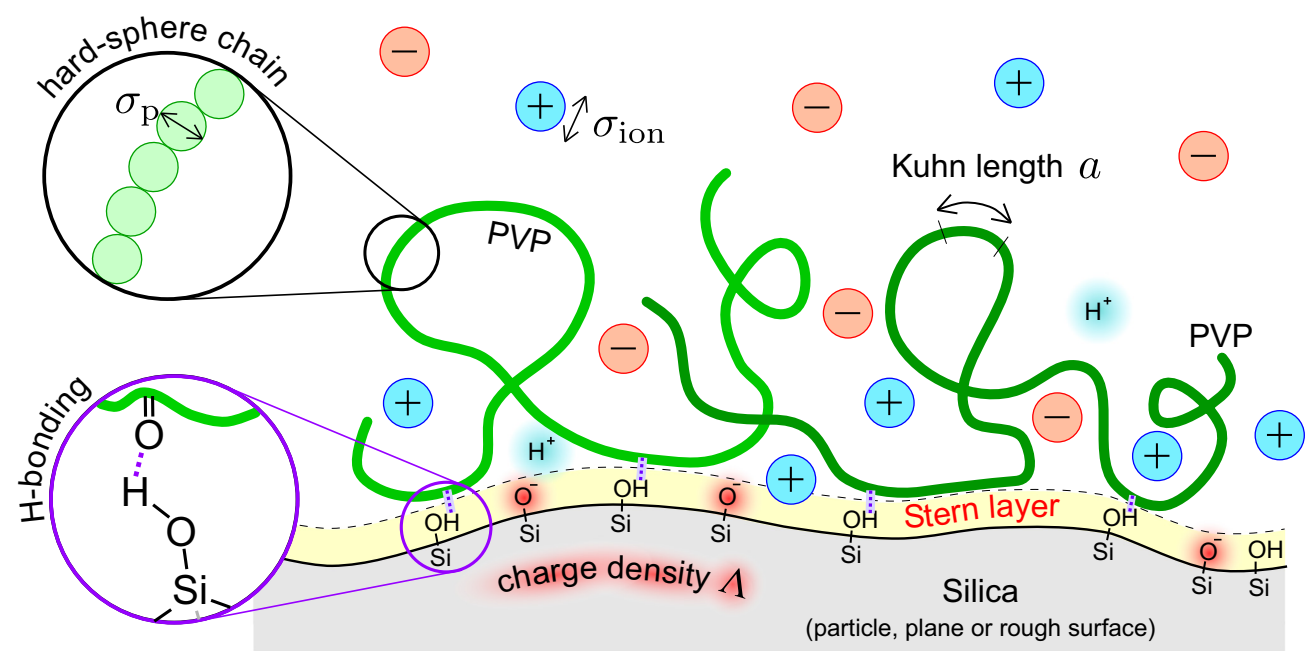

FIG. 1. (Color online) Artist's impression of the interface between a silica surface and the solution containing adsorbing polymer chains (PVP) and ions, as implemented in our model. 
can be negative (polyanions), positive (polycations), or 0 (neutral polymer chains). The polymer segments have a volume $v_{\mathrm{p}}$ per unit length (unit $\mathrm{m}^{3} / \mathrm{m}$ ). When representing the polymers as chains of connected spheres (for which the term beads will be used hereafter), we can express the bead diameter as $\sigma_{\mathrm{p}}=\sqrt{6 v_{\mathrm{p}} / \pi}$ [27]. In this way, the volume per unit length $v_{\mathrm{p}}$ of a particular polymer is related to the mass density $\rho$ of the pure polymer phase by $\rho=M /\left(v_{\mathrm{p}} d N_{\mathrm{Av}}\right)$, with $M$ the molar mass of a chemical monomer, $d$ its backbone length (e.g., about $0.25 \mathrm{~nm}$ for a $-\mathrm{CH}_{2} \mathrm{CHR}-$ backbone), and $N_{\mathrm{Av}}$ Avogadro's number.

Besides polymer chains, the solution contains positive and negative ions. For simplicity we will assume here that both ions are monovalent $\left(z_{+}=1, z_{-}=-1\right)$ and that they have the same hydrated ion size $\sigma_{\text {ion }}$. We consider an open isothermal system, in which the number density of small ions in the polymer-free electrolyte reservoir is fixed at $n_{\infty}$. Moreover, in the polymer-containing bulk solution we fix the bulk concentration of polymer to $\phi_{\mathrm{p}}^{\mathrm{b}}$ and we calculate all changes in free energy of the polymer chains with reference to this polymer bulk concentration.

When a surface is brought into contact with the polymercontaining solution, concentration gradients of polymers and ions are formed near the surface. Volume exclusion interactions between polymer chains and ions will modify the concentrations of ions near the surface. If, in addition the surface or the polymer chains, or both, are charged, electrostatic interactions also influence the concentration profiles of ions. Here we will consider a particular surface with a density of ionizable groups given by $\Lambda$ (unit $\mathrm{m}^{-2}$ ), with a charge sign $z_{\mathrm{s}}$. The surface ionization degree $\alpha_{\mathrm{s}}$ defines the fraction of ionizable groups that are charged and varies from 0 to 1 (strongly charged surfaces). For neutral surfaces $\Lambda=0$.

Solvent is implicit in our model and we treat it as a continuous medium with an average permittivity $\epsilon_{0} \epsilon_{\mathrm{w}}$ and density $\rho_{\mathrm{w}}$. Consequently, variations in the electrostatic potential or concentrations over typical distances smaller than the size of a solvent molecule $(0.28 \mathrm{~nm}$ for water) are not meaningful. However, we typically consider excluded volume interactions between hydrated ions and polymer chains, which are much larger than the individual solvent molecules. In addition, the influence of solvent molecules on directional interactions, such as hydrogen bonds, cannot be described explicitly. Instead, we describe hydrogen bonding interactions between polymer chains and a surface implicitly (see Sec. II C).

\section{A. Ground-state dominance approximation}

The density profile of a Gaussian polymer chain near a surface is described by the Edwards equation

$$
\frac{\partial G\left(r, r^{\prime}, n\right)}{\partial n}=\frac{a^{2}}{6} \nabla^{2} G\left(r, r^{\prime}, n\right)-\frac{U(r)}{k_{\mathrm{B}} T} G\left(r, r^{\prime}, n\right),
$$

where $G\left(r, r^{\prime}, N\right)$ is a two-point Green's function that is proportional to the statistical weight of a polymer chain of length $N$ with ends at positions $r$ and $r^{\prime}$ [28,29]. When applied to polymer chains, $G$ is often referred to as chain propagator or chain order parameter [28,30,31]. $U(r)$ constitutes an external potential, defined per polymer segment, which we write as $U(r) / k_{\mathrm{B}} T=a u(r)$ with $u(r)$ the exchange potential in $k_{\mathrm{B}} T$ per unit length. In our model we will adopt a mean-field approximation, implying that the exchange potential is determined by the potential of a polymer chain in a mean field of all other species. In this approximation $u(r)$ is essentially the difference between the local chemical potential (per unit length) of a polymer chain and its chemical potential in the bulk solution [32]. It includes all electrostatic, dipolar, chemical, and excess volume interactions, but not the connectivity of the polymer chains. Finally, we note that Eq. (1) implicitly assumes that the polymer chains obey random walk statistics [28,31,33,34].

The general solution to the above equation can be written as a series of eigenfunctions [29]. For very long polymer chains the contribution of the ground-state eigenfunction dominates all other terms and the solution can be limited to the ground-state term. In this so-called ground-state dominance approximation, the Edwards equation can be rewritten as

$$
\frac{a^{2}}{6} \nabla^{2} G(r)=u(r) a G(r) .
$$

The chain order parameter $G$ is related to the local polymer volume fraction $\phi_{\mathrm{p}}$ by $\phi_{\mathrm{p}}(r)=a v_{\mathrm{p}} G^{2}$ [32]. The ground-state dominance approximation is mostly applied to polymer chains bound (by adsorption, for instance) to regions with dimensions small compared to the unperturbed chain size, as the Gaussian chain statistics is likely to remain valid for sufficiently small length scales [31,34]. It has been argued that this approach cannot capture more sophisticated details of the adsorption of either neutral polymer chains or polyelectrolytes. For neutral polymer chains, the main objection is the fact that chain ends (tails) are ignored in the ground-state dominance approximation $[31,35,36]$. For polyelectrolytes, chain stiffening and charge correlations between nearby segments are not described satisfactorily [31,37]. Despite these limitations, we will use the ground-state dominance approximation in this work to provide a practicable model for the adsorption of long (possibly charged) polymer chain and to allow comparison of different adsorption mechanisms.

Before treating the exchange potential $u(r)$ in Sec. II C, we first discuss how the electrostatic potential $\psi$ is related to the concentrations of polymer chains and ions.

\section{B. Electrostatics \\ 1. Electrostatic potential}

The dimensionless electrostatic potential $y=e \psi / k_{\mathrm{B}} T$ can be obtained by solving Poisson's equation

$$
\nabla \cdot[\epsilon(r) \nabla y(r)]=-\frac{e}{k_{\mathrm{B}} T} \rho_{\mathrm{c}}(r),
$$

where $\epsilon(r)$ is the local permittivity, $e$ is the electron charge, and $\rho_{\mathrm{c}}(r)$ the local charge density. Since polymer volume fractions can become quite high near strongly adsorbing surfaces, the local permittivity $\epsilon(r)$ may be different from that of pure solvent $\epsilon_{0} \epsilon_{\mathrm{w}}$. It is therefore important to take gradients in $\epsilon$ into account in Gauss' law. Electrostatic correlations were recently included in a fourth-order modified Poisson equation [38] that could be applied to the case of polymer adsorption as well. However, we will limit our model to the second-order Poisson equation described by Eq. (3). For linear variations in local 
permittivity we can write:

$$
\epsilon(r)=\epsilon_{0}\left[\phi_{\mathrm{p}}(r) \epsilon_{\mathrm{p}}+\left(1-\phi_{\mathrm{p}}\right) \epsilon_{\mathrm{w}}\right]=\epsilon_{0} \epsilon_{\mathrm{w}}\left[1-\theta \phi_{\mathrm{p}}(r)\right],
$$

where $\epsilon_{\mathrm{w}}$ and $\epsilon_{\mathrm{p}}$ are the relative permittivities of the pure solvent (electrolyte) and the pure polymer phase, respectively, and $\theta=1-\epsilon_{\mathrm{p}} / \epsilon_{\mathrm{w}}$ is their relative difference.

The local charge density $\rho_{\mathrm{c}}(r)$ is determined by the concentration of small ions and the volume fraction of polymer chains: $\rho_{\mathrm{c}}(r) / e=z_{+} n_{+}(r)+z_{-} n_{-}(r)+\alpha_{\mathrm{p}}(r) \lambda \phi_{\mathrm{p}}(r) / v_{\mathrm{p}}$. The concentration of small ions can be written in terms of the local electrostatic potential using the modified Boltzmann equation

$$
n_{ \pm}(r)=n_{\infty} \exp \left[-z_{ \pm} y(r)-\Delta \mu_{ \pm}^{\mathrm{ex}}(r)\right],
$$

where $n_{\infty}$ is the ionic strength in the (polymer-free) reference solution and $\Delta \mu_{ \pm}^{\mathrm{ex}}(r)$ is the excess chemical potential difference per ion (in units $k_{\mathrm{B}} T$ ) due to volume exclusion between ions, and between ions and polymer segments, which we will account for using a hard-sphere approximation (see Sec. II C). Since we have assumed that both ions have the same size, $\Delta \mu_{+}^{\mathrm{ex}}=\Delta \mu_{-}^{\mathrm{ex}}=\Delta \mu_{\mathrm{ion}}^{\mathrm{ex}}$. High concentrations of ions and polymer chains close to the surface will result in strong repulsive volumetric interactions, disfavoring a further increase in concentration. The incorporation of volumetric interactions via a hard-sphere equation of state in the Poisson-Boltzmann equation has been found to yield realistic predictions for ion accumulation and surface pressure near highly charged surfaces without polymer chains [39].

Inserting Eqs. (4) and (5) in Eq. (3) yields a modified Poisson-Boltzmann equation for monovalent electrolytes:

$$
\begin{aligned}
& {\left[1-\theta \phi_{\mathrm{p}}(r)\right] \nabla^{2} y(r)-\theta \nabla \phi_{\mathrm{p}}(r) \nabla y(r)} \\
& \quad=\kappa^{2}\left(\frac{\sinh y(r)}{\exp \Delta \mu_{\mathrm{ion}}^{\text {ex }}(r)}-\frac{\phi_{\mathrm{p}}(r) \alpha_{\mathrm{p}}(r) \lambda}{2 v_{\mathrm{p}} n_{\infty}}\right),
\end{aligned}
$$

where we used the Debye screening length of the electrolyte reference solution $\kappa^{-1}$, defined by $\kappa^{2}=2 n_{\infty} e^{2} /\left(\epsilon_{0} \epsilon_{\mathrm{w}} k_{\mathrm{B}} T\right)$.

The gradient of the electrostatic potential $\nabla y(r)$ at the adsorbing surface is defined by the charge density of the surface $\Lambda$ (in $\left.\mathrm{m}^{-2}\right)$ :

$$
\left.\nabla y(r)\right|_{0}=-\frac{\alpha_{\mathrm{s}} z_{\mathrm{s}} \Lambda \kappa^{2}}{2 n_{\infty}\left(1-\theta \phi_{\mathrm{p}}^{0}\right)},
$$

where the subscript 0 at the left-hand side indicates the gradient at the surface and $\phi_{\mathrm{p}}^{0}$ at the right-hand side indicates the polymer volume fraction at the surface.

The electrostatic potential in the bulk solution $y^{\mathrm{b}}$ follows from the condition of electroneutrality. By setting the left-hand side of Eq. (6) to zero, we find

$$
y^{\mathrm{b}}=\sinh ^{-1}\left(\frac{\phi_{\mathrm{p}}^{\mathrm{b}} \alpha_{\mathrm{p}}^{\mathrm{b}} \lambda \exp \left(\Delta \mu_{\mathrm{ion}}^{\mathrm{ex}, \mathrm{b}}\right)}{2 v_{\mathrm{p}} n_{\infty}}\right),
$$

where $\Delta \mu_{\mathrm{ion}}^{\mathrm{ex}, \mathrm{b}}$ is the excess chemical potential difference per ion in the bulk (in units $k_{\mathrm{B}} T$ ) due to volume exclusion between ions (at volume fractions $\phi_{ \pm}^{\mathrm{b}}=\pi \sigma_{\text {ion }}^{3} n_{ \pm}^{\mathrm{b}} / 6$ ) and polymer segments (at volume fraction $\phi_{\mathrm{p}}^{\mathrm{b}}$ ), as compared to the isothermal ion reservoir (containing only ions at density $\left.n_{\infty}\right)$. Finally, we set the electrostatic potential gradient far from the surface to zero.

\section{Ionizable groups}

Both the polymer chains and the surface may contain (weakly) ionizable groups. In general, the degree of ionization will depend on the local electrostatic potential. For simplicity we assume only the dissociation or association of $\mathrm{H}^{+}$ions from the polymer and the surface. For PVP adsorption on silica this description suffices, and we refer to Ref. [40] for an overview of different ionization models of polymer chains and surfaces.

For the surface we can write

$$
\ln \left(\alpha_{\mathrm{s}}^{-1}-1\right)=z_{\mathrm{s}} y^{0}+z_{\mathrm{s}} \ln (10)\left(p \mathrm{H}-p K_{\mathrm{a}, \mathrm{s}}\right)
$$

and for the polymer chains

$$
\ln \left[\alpha_{\mathrm{p}}^{-1}(r)-1\right]=z_{\mathrm{p}} y(r)+z_{\mathrm{p}} \ln (10)\left(p \mathrm{H}-p K_{\mathrm{a}, \mathrm{p}}\right),
$$

where $p K_{\mathrm{a}}$ is the dissociation constant (of the polymer, $p$, or the surface, $s$ ), and $p \mathrm{H}$ is defined in the reference electrolyte solution (where $y=y_{\infty}=0$ ). In the bulk we have $y=y^{\mathrm{b}}$, and $\alpha_{\mathrm{p}}^{\mathrm{b}}$ can be calculated from Eq. (10). As a result, for ionizable polymer chains Eqs. (8) and (10) provide a closed set of expressions defining the electrostatic potential and degree of ionization in the bulk. For strongly charged polyelectrolytes, $\alpha_{\mathrm{p}}^{\mathrm{b}}=1$ and Eq. (8) defines the electrostatic potential in the bulk.

\section{Stern layer}

The fact that the ions and the polymer chains cannot approach a (charged) surface to infinite proximity due to their finite size can be taken into account by introducing the concept of a Stern layer, into which the charge of the ions and the polymer chains cannot penetrate [41]. The Stern layer has a capacitance $C_{\mathrm{St}}\left(\mathrm{F} / \mathrm{m}^{2}\right)$ and the decrease of the electrostatic potential across the Stern layer is inversely proportional to the capacitance:

$$
y^{0}-y^{d}=\frac{\alpha_{\mathrm{s}} z_{\mathrm{s}} e^{2} \Lambda}{C_{\mathrm{St}} k_{\mathrm{B}} T} .
$$

When implementing Eq. (11) for planar surfaces, Eq. (7) remains unchanged, and the surface charge $\alpha_{\mathrm{s}} z_{\mathrm{s}} \Lambda e$ is directly related to the potential at the boundary of the diffuse part of the electric double layer $y^{d}$. For strongly curved surfaces, the difference in field strength at the surface and at the boundary of the diffuse part of the electric double layer must formally be taken into account [42].

In solutions containing multiple charged species, such as cations, anions, and polyelectrolytes, the Stern layer is no longer simply a single layer of fixed capacity, as the dimensions of all charged species will be different [43]. Nevertheless, we will use the concept of a single Stern layer with fixed capacity here to account for the finite size of the small ions.

\section{Exchange potential}

The adsorption of the polymer chains is governed by the exchange potential $u(r)$ [Eq. (2)]. This exchange potential is the difference between the local chemical potential of the polymer chains (per unit length) $\mu_{\mathrm{p}}^{\mathrm{tot}}(r)$ and their chemical potential in the bulk solution $\mu_{\mathrm{p}}^{\text {tot, } \mathrm{b}}$. Here we consider the following contributions to the polymer chemical potential (all 
$\mu$ 's are expressed in $k_{\mathrm{B}} T$ per unit length, except for $\mu$ 's per bead or per ion in Sec. II C 3).

\section{Electrostatic interactions}

For polymer chains with a fixed charge, the mean-field electrostatic contribution to the chemical potential is

$$
\mu^{\mathrm{el}}=\lambda y \text {, }
$$

where $\lambda$ is the polymer line charge density. For polymer chains with weakly ionizable groups, $\lambda$ must be replaced by the actual line charge density $\alpha_{\mathrm{p}} \lambda$, and an additional (chemical) contribution for the dissociation of ionizable groups must be added to Eq. (12) [32,44]:

$$
\mu^{\mathrm{el}, \mathrm{cr}}=\alpha_{\mathrm{p}} \lambda y-z_{\mathrm{p}} \lambda \int_{0}^{\alpha_{\mathrm{p}}} y^{\prime} d \alpha_{\mathrm{p}}^{\prime}=\lambda \ln \left(1-\alpha_{\mathrm{p}}\right) .
$$

For neutral polymer chains $\lambda=0$, and there is no electrostatic contribution to the local chemical potential.

\section{Polarization}

Permittivity differences between the polymer chains $\left(\epsilon_{\mathrm{p}}\right)$ and the solution $\left(\epsilon_{\mathrm{W}}\right)$ give rise to a polarization contribution to the chemical potential in the presence of gradients of the electrostatic potential $[45,46]$ :

$$
\mu^{\text {diel }}=-\frac{v_{\mathrm{p}} k_{\mathrm{B}} T}{2 e^{2}} \frac{\partial \epsilon}{\partial \phi_{\mathrm{p}}}(\nabla y)^{2}=\frac{v_{\mathrm{p}} n_{\infty} \theta}{\kappa^{2}}(\nabla y)^{2},
$$

where we used $\left(\partial \epsilon / \partial \phi_{\mathrm{p}}\right)=-\theta \epsilon_{0} \epsilon_{\mathrm{w}}$. Gradients in $\mu^{\text {diel }}$ give rise to a dielectrophoretic force that can be used to manipulate and transport different types of particles [47].

\section{Volume exclusion}

Volume exclusion interactions between polymer chains and ions are very important in regions of high concentration, such as close to a charged, adsorbing surface. Previously, Flory-Huggins theory has been used to account for volume exclusion of polymer chains mutually $[48,49]$. Volume exclusion interactions between polymer chains and ions have been taken into account at the PB level, thus treating the ions as point charges [32]. Here, we will describe the excess chemical potential due to volume exclusion interactions using the equation of state for polydisperse mixtures of hard spheres and chains of connected spheres [50,51]. This equation of state is a modification of the well-known Boublik-Mansoori-CarnahanStarling-Leland (BMCSL) equation of state for mixtures of hard spheres. We treat the hydrated ions as charged beads with a hard-sphere diameter $\sigma_{\text {ion }}$ and the polymers as chains of connected, touching beads with a hard-sphere diameter $\sigma_{\text {pol }}$. The size of the beads that make up the polymer chains is chosen such that the chain of beads has the same volume per unit length as the polymer chain we used in the ground-state dominance approximation $\sigma_{\mathrm{p}}=\sqrt{6 v_{\mathrm{p}} / \pi}$.

In Ref. [51] we presented the modified BMCSL equation of state for such mixtures of beads and assemblies of beads, by correcting for the translational entropy of the assemblies. The excess chemical potential per bead of species $i\left(\mu_{i \text {,bead }}^{\text {ex }}\right)$ can be rewritten as the product of the excess chemical potential per unit length $\mu_{i}^{\mathrm{ex}}$, which is used in the exchange potential $u$, and the bead size $\sigma_{i}$ :

$$
\begin{aligned}
\mu_{i, \text { bead }}^{\mathrm{ex}}= & \sigma_{i} \mu_{i}^{\mathrm{ex}}=-\left(\frac{1}{N_{\mathrm{b}, i}}+\frac{2 \xi_{2}^{3} \sigma_{i}^{3}}{\phi^{3}}-\frac{3 \xi_{2}^{2} \sigma_{i}^{2}}{\phi^{2}}\right) \ln (1-\phi) \\
& +\frac{3 \xi_{2} \sigma_{i}+3 \xi_{1} \sigma_{i}{ }^{2}+\xi_{0}^{\prime} \sigma_{i}^{3}}{1-\phi}+\frac{3 \xi_{2}^{2} \sigma_{i}^{2}+3 \xi_{1} \xi_{2} \sigma_{i}^{3} \phi}{\phi(1-\phi)^{2}} \\
& -\xi_{2}^{3} \sigma_{i}{ }^{3}\left(\frac{\phi^{2}-5 \phi+2}{\phi^{2}(1-\phi)^{3}}\right),
\end{aligned}
$$

where the $\xi_{k}$ 's are weighted sums of the volume fractions of all species, which depend on the position in solution: $\xi_{k}=\sum_{j} \phi_{j} \sigma_{j}^{k-3}$, and $\xi_{0}^{\prime}=\sum_{j} \phi_{j} \sigma_{j}^{-3} N_{\mathrm{b}, j}^{-1}$. The $\sigma_{i}$ 's are the (effective hard-sphere) diameters of the beads of species $i$, $N_{\mathrm{b}, \mathrm{p}}=L / \sigma_{\mathrm{p}}$ is the number of connected beads per polymer chain, and $\phi$ is the total volume fraction of all species: $\phi=\sum_{j} \phi_{j}$. For the solution of polymer chains and ions we consider here, $\phi=\phi_{\mathrm{p}}+\phi_{+}+\phi_{-}$.

Equation (15) is generally valid for arbitrary mixtures of long and short polymer chains, and ions of various sizes. This equation can be used for both the ions $\left(N_{\mathrm{b}, \text { ion }}=1\right)$ and the polymer chains $\left(N_{\mathrm{b}, \mathrm{p}}=L / \sigma_{\mathrm{p}}\right)$, and can be expanded into a power series of the total volume fraction $\phi$, resulting in Eq. (7.3) of Ref. [51].

Elegant simplifications of Eq. (15) exists in a number of well-studied limits: (i) a solution of only monomers (or ions), each having a hard-sphere diameter $\sigma$, (ii) a solution of monomers (or ions) of size $\sigma_{\mathrm{p}}$ and polymer chains (of connected monomeric beads of the same size), and (iii) a solution of polymer chains with ions that are treated as point charges $\left(\sigma_{\text {ion }}=0\right)$.

In situation (i), the excess chemical potential (per monomer or per ion) is given by the well-known Carnahan-Starling equation:

$$
\mu_{\mathrm{ion}, \mathrm{CS}}^{\mathrm{ex}}=\frac{\phi\left(8-9 \phi+3 \phi^{2}\right)}{(1-\phi)^{3}}=\frac{3-\phi}{(1-\phi)^{3}}-3
$$

with $\phi$ the total monomer or ion volume fraction.

In situation (ii), taking $N_{\mathrm{b}, \mathrm{p}} \rightarrow \infty$, the excess chemical potential of the polymer chains (per polymeric bead) simplifies to a modified Carnahan-Starling equation:

$$
\mu_{\mathrm{pol}, \mathrm{CS}}^{\mathrm{ex}}=\ln (1-\phi)+\frac{f_{\mathrm{m}} \phi}{1-\phi}+\frac{7 \phi}{(1-\phi)^{2}}+\frac{2 \phi^{3}}{(1-\phi)^{3}}
$$

with $f_{\mathrm{m}}$ the fraction of all beads that are not part of the polymer chains. If the solution contains only polymers, $f_{\mathrm{m}}=0$ and the second term in Eq. (17) vanishes, leading to a well-known power series expansion [27,57]:

$$
\mu_{\mathrm{pol}, \mathrm{CS}}^{\mathrm{ex}}=6 \phi_{\mathrm{p}}+\frac{27}{2} \phi_{\mathrm{p}}^{2}+\frac{68}{3} \phi_{\mathrm{p}}^{3}+\ldots
$$

The excess chemical potential of the monomeric beads in this mixture is slightly higher, because of their translational entropy:

$$
\mu_{\mathrm{mon}, \mathrm{CS}}^{\mathrm{ex}}=\frac{f_{\mathrm{m}} \phi}{1-\phi}+\frac{7 \phi}{(1-\phi)^{2}}+\frac{2 \phi^{3}}{(1-\phi)^{3}} .
$$

If ions of negligible volume are added to such a polymer solution [situation (iii)], an ion insertion pressure $v_{\mathrm{p}} \sigma_{\mathrm{p}} \Pi$ must be added to the polymer chemical potential, where 
$\Pi=n_{\infty} /\left(1-\phi_{\mathrm{p}}\right)$ is the osmotic pressure due to the ions [51] For the ionic point charges, an excess chemical potential due to volume exclusion by the polymer chains can be found from Eq. (15) (with $\sigma_{\text {ion }}=0$ and $N_{\mathrm{b}, \mathrm{p}} \rightarrow \infty$ ):

$$
\mu_{\text {ions, point }}^{\mathrm{ex}}=-\ln (1-\phi) .
$$

Returning to the general case of a mixture of polymer chains and ions, which both have a nonzero effective hard core size, we will use the full Eq. (15) for both the polymer chains and the ions in our model. For the ions we insert the excess chemical potential difference per ion (per bead), $\mu_{\text {ion,bead }}^{\text {ex }}$, in the Boltzmann equation [Eqs. (5) and (8), abbreviated there as $\left.\mu_{\text {ion }}^{\text {ex }}\right]$, using the ion reservoir (with $\phi_{\text {ion }}=2 \pi \sigma_{\text {ion }}^{3} n_{\infty} / 6$ ) as a reference. For the polymer chains, we include the excess chemical potential per unit length $\mu_{\mathrm{p}}^{\mathrm{ex}}$ in the exchange potential $u(r)$.

\section{Ideal mixing entropy}

An ideal entropy contribution to the exchange potential of the polymer chains is generally omitted in the ground state dominance approximation, as this approach is valid only for sufficiently long chains $(N \rightarrow \infty)$ and the ideal entropy contribution to the exchange potential scales as $1 / N$ per unit length:

$$
\mu^{\mathrm{id}}=\frac{\ln \phi_{\mathrm{p}}}{L} .
$$

For ions, however, the ideal entropy term is crucial, and is included in the Boltzmann equation (5).

\section{Chemical interactions between polymer chains}

Nonelectrostatic attractions or repulsions that are not related to volume exclusions between the polymer chains (e.g., hydrophobic interactions) can be included in the same way as in Flory-Huggins theory:

$$
\mu^{\text {att }}=-2 \chi v_{\mathrm{p}} \phi_{\mathrm{p}},
$$

where $\chi$ is an energy per unit volume of the polymer chains, and $\chi v_{\mathrm{p}}$ is the corresponding energy per unit length. A positive energy implies attraction between the polymer chains.

\section{Chemical interactions of polymers with the surface}

Besides electrostatic attractions between polymer chains and the surface, adsorption of polymer chains can be driven by a physical interaction (e.g., Van der Waals force between the polymer chains and the surface) or by the formation of specific (chemical) bonds between polymer chains and surface (e.g., $\mathrm{H}$ bonds). We are specifically interested in describing $\mathrm{H}$-bond interactions between the surface and the polymer chains and we take these interactions into account by assuming that the range of H-bonding interactions is very short, and that the decay of the adsorption potential near the surface as a result of $\mathrm{H}$ bonds can be described in the following general way:

$$
\mu^{\text {ads }}(x)=\varepsilon_{\mathrm{s}} \exp \left[-\omega\left(x / \sigma_{\mathrm{p}}+\delta\right)^{6}\right]
$$

The parameters $\omega$ and $\delta$ affect the range of attraction beyond the bead size $\sigma_{\mathrm{p}}$ : the adsorption potential has decreased to half its value at the surface at $x_{1 / 2}=\sigma_{\mathrm{p}} \ln (2) / \omega-\delta \sigma_{\mathrm{p}}$. In addition, $\omega$ also affects the sharpness of the decay: $\partial \mu^{\text {ads }} /\left.\partial x\right|_{1 / 2} \approx$ $-\varepsilon_{\mathrm{s}} /\left(\sigma_{\mathrm{p}} \omega^{4}\right)$. We typically use $\omega=0.5$ and $\delta=0$, such that the adsorption potential decays sharply to 0 around $r=\sigma_{\mathrm{p}}$, implying that only polymer segments that are closer than the effective bead size will be attracted by the surface.

The adsorption strength per unit length $\varepsilon_{\mathrm{s}}$ can be adapted to the nature of the polymer-surface interactions: a fixed value $\varepsilon_{\mathrm{s}}\left(k_{\mathrm{B}} T\right.$ per unit length) for hydrophobic interactions, or proportional to the number of surface $\mathrm{OH}$ groups for $\mathrm{H}$ bonds: $\varepsilon_{\mathrm{s}}=\varepsilon_{\mathrm{s}}^{\prime}\left(1-\alpha_{\mathrm{s}}\right) \Lambda$.

In previous work on adsorption of neutral polymer chains and polyelectrolytes where the size of the polymer chains and the ions was not explicitly taken into account, the adsorption potential was assumed to be an infinitely sharp function at the surface [28-30]. Our choice of the adsorption potential in Eq. (23) ensures a narrow adsorption region (i.e., the adsorption layer is small compared to the unperturbed chain size), while allowing easy numerical implementation.

\section{Combined}

The exchange potential per unit length $u$ that governs the adsorption of polymer chains is a combination of the different contributions listed above, taking into account the reference values in the bulk, where $\phi_{\mathrm{p}}=\phi_{\mathrm{p}}^{\mathrm{b}}, y=y^{\mathrm{b}}, \alpha_{\mathrm{p}}=\alpha_{\mathrm{p}}^{\mathrm{b}}, \phi_{+}=$ $\pi \sigma_{\text {ion }}^{3} n_{+}^{\mathrm{b}} / 6, \phi_{-}=\pi \sigma_{\text {ion }}^{3} n_{-}^{\mathrm{b}} / 6$, and $n_{+}^{\mathrm{b}}$ and $n_{-}^{\mathrm{b}}$ are given by Eq. (5) (with $\Delta \mu_{\text {ion }}^{\text {ex,b }}$ of the bulk).

In the following sections we will consider the adsorption of polymer chains to flat surfaces. Therefore, we only need to take into account concentration gradients perpendicular to the surface (in a direction we call $x$ ). The boundary conditions for the key variables $G$ and $y$ are set as follows. At the surface, $d G / d x=0$ (implying an indifferent surface [32,52]) and $d y / d x$ is given by Eq. (7). In the bulk, $d G / d x=0$ and $d y / d x=0$.

We solved the adsorption profiles of polymer chains and the corresponding concentration profiles of ions numerically, adopting a finite difference scheme to approximate all derivatives. We use a simple linear discretization with a typical step of $0.05 \mathrm{~nm}$, and we verified that finer discretization yields the same adsorbed amounts.

The adsorbed amount of polymer (mass per unit area) is calculated from:

$$
\Gamma=\rho \int_{0}^{\infty}\left[\phi_{\mathrm{p}}(r)-\phi_{\mathrm{p}}^{\mathrm{b}}\right] d x,
$$

where $\rho=M /\left(v_{\mathrm{p}} d N_{\mathrm{Av}}\right)$ is the mass density of the pure polymer $\left(\rho=1.2 \mathrm{~g} / \mathrm{cm}^{3}\right.$ for PVP, and we use $v_{\mathrm{p}}=0.67 \mathrm{~nm}^{3} / \mathrm{nm}$ and $d=0.25 \mathrm{~nm})$.

Before concentrating further on the specific case of PVP adsorption to silica surfaces, we examined the importance of excluded volume interactions for a typical case of polyelectrolyte chains adsorbing to an oppositely charged surface with a fixed surface charge in the presence of ions. Figure 2 shows how the adsorption profile of polyelectrolytes and the ion density profile change as we take excluded volume interactions between ions and polymer chains into account at different levels. Profiles I-IV correspond to the profiles reported in Fig. 1 of Ref. [32], with IV including volume exclusion by the ions approximated via the osmotic pressure, and profile $\mathrm{V}$ was calculated using the modified BMCSL equation of state given 

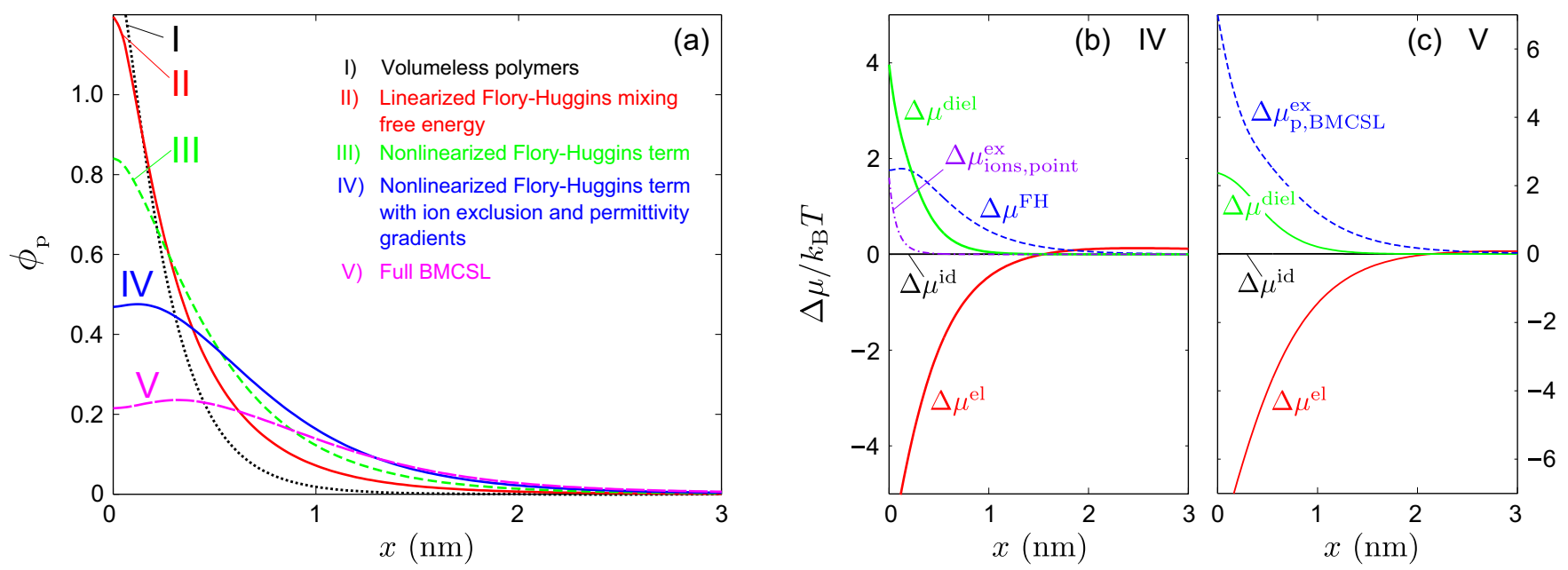

FIG. 2. (Color online) (a) Adsorption profiles of polyelectrolytes on an oppositely charged surface in the presence of a 1:1 electrolyte, using various models to account for the excluded volume interactions. Profiles I-IV are calculated using the exchange potential definitions in Fig. 1 of Ref. [32] $\left(v=0.5 \mathrm{~nm}^{3} / \mathrm{nm}, v_{\mathrm{FH}}=0.18 \mathrm{~nm}^{3}, z_{\mathrm{s}} \Lambda=-1 \mathrm{~nm}^{-2}\right.$ (fixed charge), $z_{\mathrm{p}} \lambda=1 \mathrm{~nm}^{-1}$ (fixed charge), $a=1 \mathrm{~nm}, \epsilon_{\mathrm{p}}=20$, $\epsilon_{\mathrm{w}}=78, \chi=0, n_{\infty} / N_{\mathrm{Av}}=10 \mathrm{mM}$, and $\left.\phi_{\mathrm{p}}^{\mathrm{b}}=10^{-3}\right)$, profile $\mathrm{V}$ includes the volume exclusion term of Eq. (15) for both polymer chains and ions $\left(\sigma_{\mathrm{p}}=\sqrt{6 v / \pi}=0.98 \mathrm{~nm}\right.$ and $\sigma_{\mathrm{ion}}=0.69 \mathrm{~nm}$ ), and $x$ denotes the distance from the surface. (b) and (c) Individual contributions to the exchange potential $u$ for profile IV and V, respectively. All potential energy differences approach zero far from the surface. The electrostatic potential difference $\Delta \mu^{\mathrm{el}}$ first becomes positive (around $x \approx 2 \mathrm{~nm}$ ) before decaying to zero.

by Eq. (15) to account for all excluded volume interactions. The relative contributions to the exchange potential $u$ for profiles IV and V are plotted in Figs. 2(b) and 2(c), respectively. It is clear that volume exclusion $\left(\Delta \mu^{\mathrm{FH}}+\Delta \mu_{\text {ions, points }}^{\text {ex }}\right.$ or $\left.\Delta \mu_{\mathrm{p}, \mathrm{BMCSL}}^{\mathrm{ex}}\right)$ is the dominant repulsive interaction, limiting the accumulation of polymer chains (and ions) close to the surface. When taking these excluded volume interactions into account using the modified BMCSL equation of state, we find an adsorbed amount of $0.4 \mathrm{mg} / \mathrm{m}^{2}$ (for $\rho=1.2 \mathrm{~g} / \mathrm{cm}^{3}$ ), which is typical for the adsorption of hydrophilic polyelectrolytes on oppositely charged surfaces [53].

\section{RESULTS AND DISCUSSION}

We further tested the continuum model described above by calculating the $p \mathrm{H}$ and salt-dependent adsorption isotherms for the classical experimental model system of PVP and silica. The adsorption experiments were carried out with aqueous solutions of PVP $\left(M_{\mathrm{w}}=40 \mathrm{~kg} / \mathrm{mol}\right.$, PolyScience, USA) on silicon wafers with a thermally grown silicon oxide layer $(75 \mathrm{~nm})$ that were freshly cleaned directly before the experiments using either a piranha solution or an oxygen plasma. The experimental procedures are described in detail in Ref. [25]. Plasma-cleaned surfaces exhibited a static water (Milli-Q) contact angle $\theta \approx 30^{\circ}$, whereas piranha-cleaned surfaces had a static contact angle $\theta<10^{\circ}$. Adsorption isotherms were recorded using a fixed angle optical reflectometer, equipped with an impinging jet flow cell [15].

In our model we describe the PVP chains with a Kuhn length $a$ of $1 \mathrm{~nm}$ and a volume $v_{\mathrm{p}}$ of $0.67 \mathrm{~nm}^{3} / \mathrm{nm}$, which corresponds to a pure polymer mass density $\rho=1.2 \mathrm{~g} / \mathrm{cm}^{3}$. To implement excluded volume interactions, the polymer chains will be modeled by an equivalent chain of connected beads of size $\sigma_{\mathrm{p}}=1.13 \mathrm{~nm}$ each. The polymer chains are neutral $(\lambda=0)$, and we assume there are no nonelectrostatic attractions or repulsions (other than hard-sphere volume exclusion) between the polymer chains: $\chi=0$, hence $\mu^{\text {att }}=0$ [Eq. (22)]. Unless specified otherwise we set $\phi_{\mathrm{p}}^{\mathrm{b}}=10^{-3}$, which would correspond to an equilibrium bulk PVP concentration of $1.2 \mathrm{~g} / \mathrm{L}$.

Both monovalent ions $\left(z_{+}=1, z_{-}=-1\right)$ are described as hard spheres with a hydrated diameter $\sigma_{\text {ion }}=0.69 \mathrm{~nm}$, corresponding to the average hydrated diameter of $\mathrm{Na}^{+}(0.716$ $\mathrm{nm})$ and $\mathrm{Cl}^{-}(0.664 \mathrm{~nm})$ [54].

We model the silica surface as a flat surface with ionizable SiOH groups $\left(z_{\mathrm{s}}=-1\right)$ at a total density $\Lambda$. The dissociation equilibrium of $\mathrm{SiOH}$ groups can be modeled as a single step reaction with a $p K_{\mathrm{a}, \mathrm{s}}=7.5$ [55]. As will become clear, the total density of ionizable groups $\Lambda$ depends on the pretreatment of the surface and is a crucial parameter in determining the adsorption behavior. For amorphous layers of silicon dioxide [56], the density $\Lambda$ has an upper limit of $5 \mathrm{~nm}^{-2}$ [22,24]. For piranha-cleaned surfaces we used $\Lambda=3.8 \mathrm{~nm}^{-2}$, whereas for plasma-cleaned silica surfaces we used $\Lambda=1.5 \mathrm{~nm}^{-2}$ to fit the adsorption isotherms to the data.

\section{A. Hydrogen bonding and the effects of $p H$}

The hydrogen bonding between the PVP and the $\mathrm{SiOH}$ surface groups are included using the adsorption potential in Eq. (23), with an adsorption strength that is proportional to the potential density of $\mathrm{H}$ bonds. This potential density of $\mathrm{H}$ bonds is given by the actual density of $\mathrm{SiOH}$ groups $\left(1-\alpha_{\mathrm{s}}\right) \Lambda$, with an upper limit that we describe empirically as $\sigma_{\mathrm{p}} /\left(3 v_{\mathrm{p}} d\right)$ (in $\mathrm{nm}^{-2}$ ), originating from the maximum number of $\mathrm{H}$-bond acceptors on PVP $\left[\pi \sigma_{\mathrm{p}}^{3} /\left(12 v_{\mathrm{p}} d\right)\right.$ per half a bead], divided by the projected area $\left(\pi \sigma_{\mathrm{p}}^{2} / 4\right)$. The strength of the adsorption is dictated by the amplitude $\varepsilon_{\mathrm{s}}^{\prime}$ of the adsorption potential and we used a value of $-\varepsilon_{\mathrm{s}}^{\prime} / k_{\mathrm{B}} T=2.3 \mathrm{~nm}$ [Eq. (23)], which amounts 

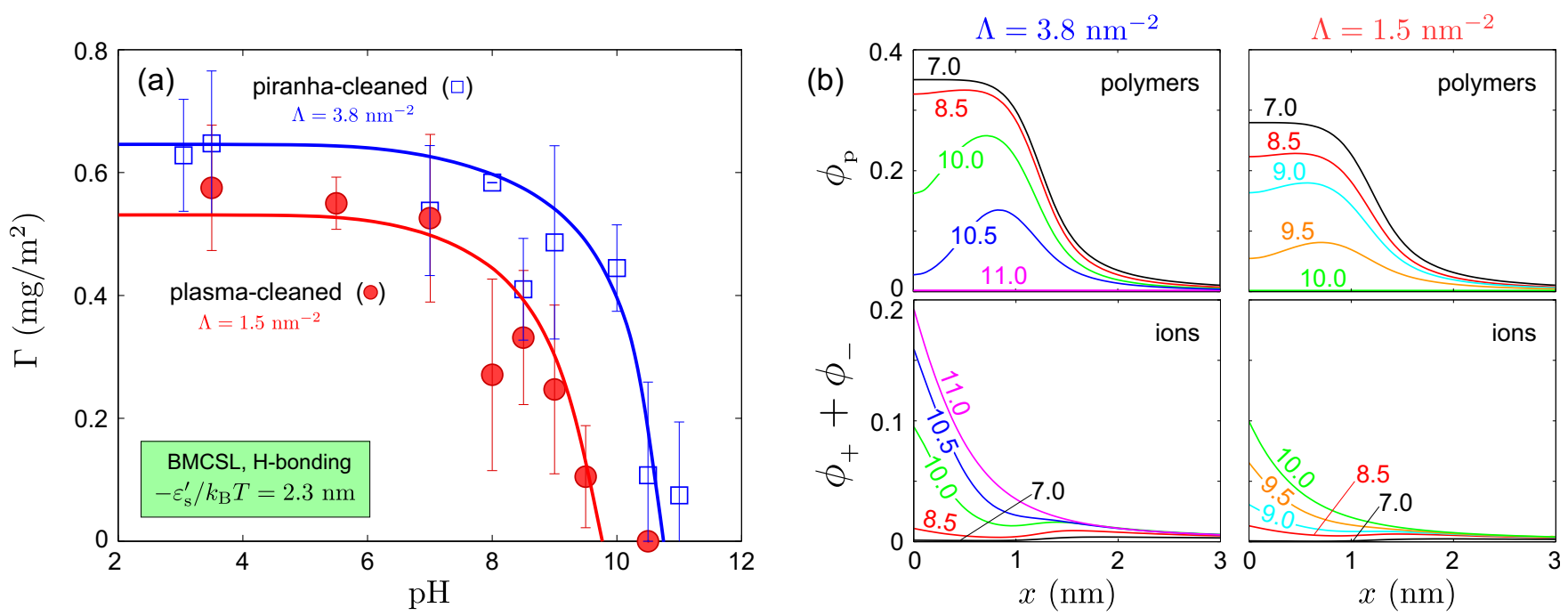

FIG. 3. (Color online) (a) Adsorbed amount of PVP on silica as a function of the solution bulk $p \mathrm{H}$. The adsorption strength is assumed proportional to the number of potential $\mathrm{H}$ bonds between PVP and silica [Eq. (23), with $\varepsilon_{\mathrm{s}}^{\prime}=-3.5 k_{\mathrm{B}} T / \mathrm{nm}$, and $\Lambda=3.8 \mathrm{~nm}{ }^{-2}$ for piranhacleaned surfaces and $1.5 \mathrm{~nm}^{-2}$ for plasma-cleaned surfaces]. The Stern layer capacitance is $C_{\mathrm{St}}=2.4 \mathrm{~F} / \mathrm{m}^{2}$. (b) Polymer (top) and ion (bottom) volume fraction profiles near silica surfaces with $\Lambda=3.8$ (left) and 1.5 (right) $\mathrm{nm}^{-2}$, at varying solution $p \mathrm{H}$ as indicated by the labels.

to a maximum adsorption energy (per unit length of polymer) of about $5.2 k_{\mathrm{B}} T / \mathrm{nm}$, based on the maximum potential $\mathrm{H}$-bond density described above. If we further assume that $d=0.25 \mathrm{~nm}$ per monomeric unit, we find a typical $\mathrm{H}$-bonding energy of $1.3 k_{\mathrm{B}} T$ per segment. Cohen Stuart et al. have reported a segmental adsorption energy of $\chi=4 k_{\mathrm{B}} T$, based on analysis of experimental displacement data in the context of a lattice-based theory [14]. This value is significantly larger than the value we have used. A full comparison is, however, difficult, because in our case $\varepsilon_{\mathrm{s}}^{\prime}$ defines only the amplitude of the adsorption potential, and the finite range of the potential, which is of order $\sigma_{\mathrm{p}}$ in our case, also affects the adsorbed amount.

Figure 3 shows the experimental and theoretical polymer adsorption as a function of $p \mathrm{H}$ for the two types of silica surfaces. The main difference between the two experimental adsorption isotherms is a shift in the $p \mathrm{H}$ value at which the adsorbed amount of polymer sharply decreases, by about $1 \mathrm{pH}$ unit. This seemingly small difference is significant, because in that region the driving force for dissociation of surface groups increases by a factor of 10 [Eq. (9)], assuming that the $p K_{\mathrm{a}, \mathrm{s}}$ of the surface groups does not change. Our model correctly describes the adsorption to both types of silica surfaces with only the density of ionizable groups $\Lambda$ as an adjustable parameter. This is in accordance with the hypothesis derived from contact angle measurements that the difference in cleaning procedure leads to different densities of $\mathrm{SiOH}$ groups on the surface [25]: a plasma-cleaned surface will have mostly siloxane groups ( $\mathrm{Si}-\mathrm{O}-\mathrm{Si}$ ) at the silicon-air interface, whereas a piranha-cleaned surface has (up to four times) more silanol groups $(\mathrm{SiOH})$.

We note that the difference in silanol density does not lead to a large difference in adsorbed amount at low $p \mathrm{H}$. Both on piranha- and on plasma-cleaned surfaces PVP is found to adsorb at $0.6 \mathrm{mg} / \mathrm{m}^{2}$ in experiments, which is well captured by our model of hydrogen bonding. The reason for the identical adsorbed amount is that the capacity for hydrogen bond formation is limited from two sides, by the hydrogen bond donors $(\mathrm{SiOH})$ on one side, and by acceptors (PVP) on the other. At low $p \mathrm{H}$, the density of hydrogen bond donors on silica is larger than the maximum acceptor density and the contribution of hydrogen bonds to the exchange potential is almost equal for both surfaces at low $p \mathrm{H}$.

As a direct consequence of the different $\mathrm{SiOH}$ surface densities, Fig. 3(b) shows that the polymer density profiles near plasma- and piranha-cleaned surfaces are qualitatively different around the transition points. Piranha-cleaned silica surfaces have a sufficiently high density of $\mathrm{SiOH}$ groups that PVP can still adsorb at relatively high degrees of dissociation $(p \mathrm{H} \sim 10)$. However, the significant accumulation of ions close to the charged surface leads to strong volumetric repulsion and expulsion of polymer segments from the regions of highest ion density, giving rise to a distinct peak in polymer density at a characteristic distance of the order of the ion size [see Fig. 3(b)]. On plasma-cleaned surfaces the density of $\mathrm{SiOH}$ groups is lower and volume exclusion is not yet significant at the transition $p \mathrm{H}$. Therefore, polymer density profiles remain sigmoidal with the maximum density closest to the surface. This difference is likely measurable in neutron reflection experiments for instance, and could be relevant for systems in which polymer chains contribute to stabilization against aggregation.

To further investigate the nature of the adsorption between PVP and silica, we adapted our model for the driving force for adsorption. We implemented either a fixed adsorption strength $\left[-\varepsilon_{\mathrm{s}}=4.0 k_{\mathrm{B}} T / \mathrm{nm}\right.$ in Eq. (23)] or a hypothetical protonation of PVP, yielding a charge-regulated electrostatic attraction between the silica surface and PVP $\left[\right.$ with $\lambda=4 \mathrm{~nm}^{-1}, p K_{\mathrm{a}, \mathrm{p}}=$ 6 in Eq. (13) and $\varepsilon_{\mathrm{s}}=0$ ]. Figure 4(a) shows the best possible fits for both alternative adsorption mechanisms. Clearly, both alternatives fail to predict the correct experimental adsorption isotherm for PVP on silica. The first alternative is that of a fixed adsorption strength, which is often assumed for hydrophobic polymers and hydrophobic surfaces, and fails to predict the 

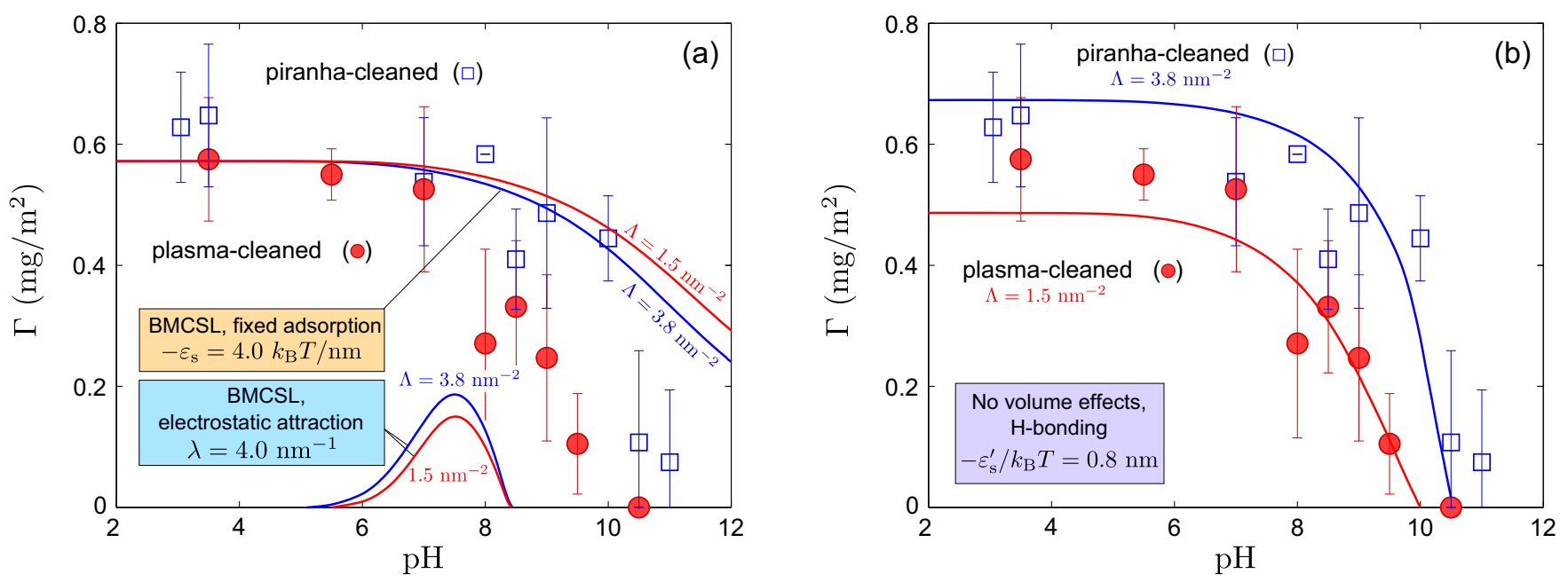

FIG. 4. (Color online) Alternative models for the adsorbed amount of PVP on silica as a function of the solution bulk $p \mathrm{H}$ : (a) fixed adsorption strength of $-4 k_{\mathrm{B}} T$ per unit length of polymer, regardless the number of Si-OH groups on the surface (dashed lines), and electrostatic attraction between the ionizable silica surface and the polymer chains $\left(\lambda=4.0 \mathrm{~nm}^{-1}, \mathrm{p} K_{\mathrm{a}, \mathrm{p}}=6\right.$, dotted lines); (b) $\mathrm{H}$ bonding without volume effects, using an adsorption strength $\varepsilon_{\mathrm{s}}^{\prime}=-1.2 k_{\mathrm{B}} T / \mathrm{nm}$ [Eq. (23)]. In both cases, the density of ionizable groups on the silica surface is $\Lambda=3.8$ (piranha-cleaned) and $1.5 \mathrm{~nm}^{-2}$ (plasma-cleaned).

very sharp desorption transition around $p \mathrm{H} 9$ (plasma-cleaned) or $p \mathrm{H} 10$ (piranha-cleaned). The second alternative of an electrostatic attraction yields a completely different shape of the $p \mathrm{H}$-dependent adsorption isotherm. The adsorbed amount goes to zero at extreme $p \mathrm{H}$, as the driving force for adsorption vanishes at low $p \mathrm{H}$ (surface uncharged) and high $p \mathrm{H}$ (polymer uncharged). Moreover, the adsorbed amount can only be increased significantly if the $p K_{\mathrm{a}, \mathrm{p}}$ of the polymeric groups is taken much higher than that of the silanol groups, which would mean that the polymer chains are strongly positively charged up to $p \mathrm{H} 7$, which is experimentally not the case for PVP.

We finally assess the relevance of excluded volume interactions in our model by calculating the same adsorption profiles without any volume exclusion [see Fig. 4(b)]. In this case, the desorption transition at $p \mathrm{H}>p K_{\mathrm{a}, \mathrm{s}}$ becomes less sharp, especially for the plasma-cleaned surfaces. Secondly, the adsorbed amount at low $p \mathrm{H}$ is predicted to be slightly lower for the plasma-cleaned surface, and we underestimate systematically the experimental data at low $p \mathrm{H}$. Finally, to fit the experimental data the $\mathrm{H}$-bonding adsorption strength must be decreased to unrealistically low values $\left(-\varepsilon_{\mathrm{s}}^{\prime} / k_{\mathrm{B}} T=\right.$ $1.8 \mathrm{~nm}$, or $\sim 0.46 k_{\mathrm{B}} T$ per $\mathrm{H}$ bond). We thus conclude that both the hydrogen bonding-based adsorption mechanism [Fig. 4(a)] and the excluded volume interactions between ions and polymer chains [Fig. 4(b)] are important to include, in order to correctly predict the adsorption isotherms of PVP to silica.

\section{B. Salt-induced desorption of polymer}

Apart from the solution $p \mathrm{H}$, adsorption of PVP to silica is affected strongly by the bulk ionic strength. This can be understood qualitatively by the fact that ions can screen surface charges of the silica, leading to enhanced proton dissociation and, hence, a lower density of $\mathrm{SiOH}$ groups and a weaker hydrogen bond-based adsorption. Experiments indicate that the adsorbed amount of PVP can be decreased to zero at $p \mathrm{H} 7$ by increasing the bulk concentration of monovalent electrolyte to $1 \mathrm{M}$. At low $p \mathrm{H}$ the adsorption does not decrease upon increasing the ionic strength from $1 \mathrm{mM}$ to $1 \mathrm{M}$, since the driving force for dissociation is negligible [Eq. (9)]. In the field of polymer adsorption, the effect of ionic strength has not yet been described by off-lattice models [26]. In SCF lattice models, the desorption transition could be shifted to lower $p \mathrm{H}$ by increasing the salt concentration, but not below the $p K_{\mathrm{a}, \mathrm{s}}$ of the silica surface [24]. Here, we investigate the salt dependence of polymer adsorption in our off-lattice model.

Figure 5 shows the experimental data and model prediction for the adsorbed amount as a function of bulk ionic strength. At $p \mathrm{H} 3.5$ no significant change in the adsorbed amount was measured for salt concentrations between $1 \mathrm{mM}$ and $1 \mathrm{M}$. At $p \mathrm{H} \mathrm{7,} \mathrm{however,} \mathrm{the} \mathrm{adsorbed} \mathrm{amount} \mathrm{decreased} \mathrm{from} \mathrm{close}$ to $0.6 \mathrm{mg} / \mathrm{m}^{2}$ at $1 \mathrm{mM} \mathrm{NaCl}$ to $0 \mathrm{mg} / \mathrm{m}^{2}$ at $1 \mathrm{M} \mathrm{NaCl}$. Our model predicts a similar salt-induced desorption, but only at significantly higher $p \mathrm{H}$ than found experimentally. At $p \mathrm{H} 7$ we find no desorption, but instead a slightly increased adsorption at very high salt concentrations, due to the fact the the bulk ion volume fraction increases to non-negligible values and therefore the difference in chemical potential $\left(\mu_{\mathrm{p}, \mathrm{BMCSL}}^{\mathrm{ex}}\right)$ between the bulk and the surface becomes smaller [see Fig. 5(b)]. At $p \mathrm{H} 8.5$, however, we do predict full desorption from an initial $\Gamma \approx 0.5 \mathrm{mg} / \mathrm{m}^{2}$ upon increasing the ionic strength to $1 \mathrm{M}$. These findings are very similar to those of Postmus et al. for lattice-based models of polymer adsorption [24]. They also found that the anticipated effect of ions, to screen the silica surface charge and increase the dissociation of silanol groups, only occurs for $p \mathrm{H}>p K_{\mathrm{a}, \mathrm{s}}$, because there is hardly any silanol dissociation at low $p \mathrm{H}$. Thus, the remarkable dissociation at $p \mathrm{H} 7$ in experiments, which has been observed before in experiments of PEO adsorption on silica [24], is therefore likely caused by additional interactions between the ions and the surface. One possibility, as suggested by Postmus et al., is that specific chemical interactions between 

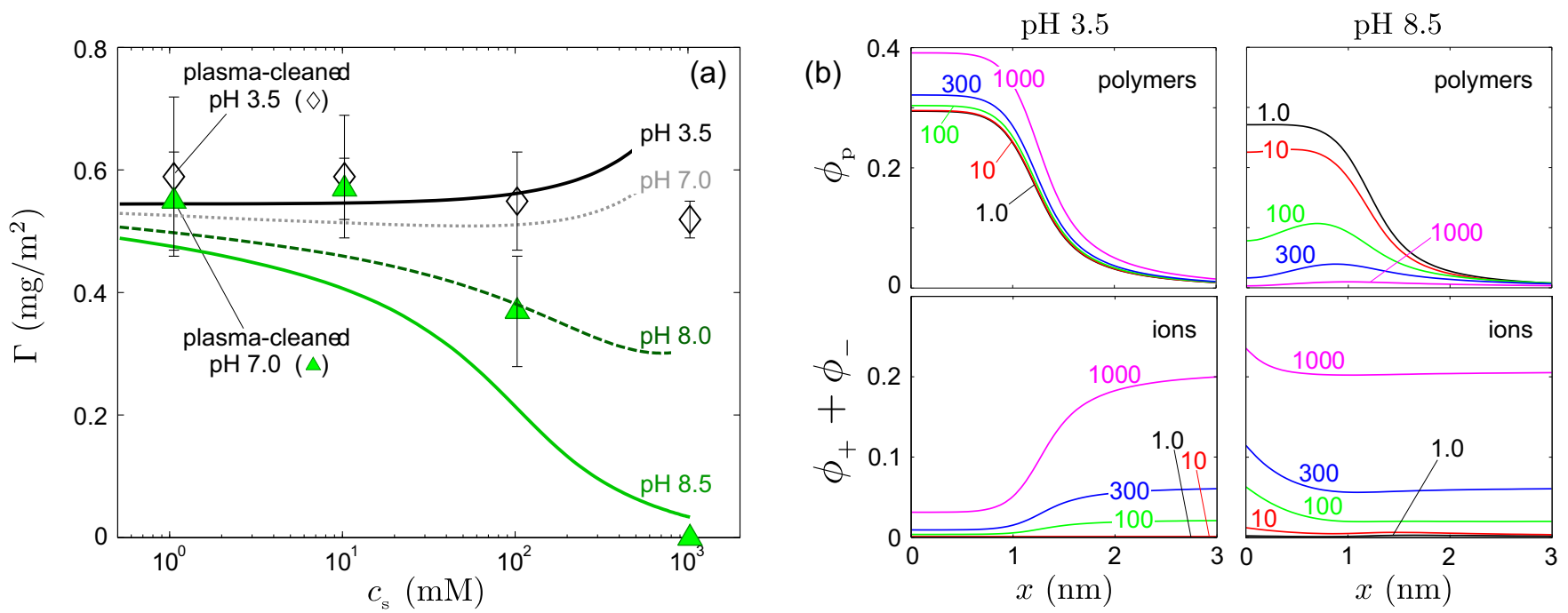

FIG. 5. (Color online) (a) Adsorbed amount of PVP on plasma-cleaned silica as a function of the bulk ionic strength for two $p \mathrm{H}$ values in experiments: $p \mathrm{H} 3.5$ (open diamonds) and 7.0 (filled triangles). The predicted salt-dependent adsorption isotherms of our model as described in relation to Fig. 3 are shown for four $p \mathrm{H}$ values (3.5, 7.0, 8.0, and 8.5, as indicated by the labels). (b) Polymer (top) and ion (bottom) volume fraction profiles near plasma-cleaned silica surfaces at $p \mathrm{H} 3.5$ (left) and 8.5 (right), at ionic strength as indicated by the labels.

the (uncharged) silica and the counterions lead to an enhanced counterion density at the silica surface and a decreased accessibility of H-bond donors and, hence, a lower adsorbed amount.

\section{CONCLUSIONS}

We have developed an off-lattice (continuum) model to describe the adsorption of neutral polymer chains and polyelectrolytes to surfaces. Our model includes excluded volume interactions between polymer chains and ions by means of a hard-sphere equation of state of mixtures of beads and strings of connected beads. For a set of experiments on the PVP/silica model system, we could correctly predict the adsorption isotherms for plasma-cleaned and piranha-cleaned surfaces, taking into account that the only change induced by the cleaning technique is the density of silanol groups. The driving force for adsorption was found to be the formation of hydrogen bonds (1.3 $k_{\mathrm{B}} T$ per hydrogen bond), which is limited by both the density of silanol groups and the density of PVP chains. Alternative models, in which the adsorption energy is constant or driven by electrostatic attraction all fail to describe the experimental adsorption isotherms. Our model was able to predict a salt-induced desorption at $p \mathrm{H}>p K_{\mathrm{a}, \mathrm{s}}$, but not at $p \mathrm{H}$ as low as 7, which was found experimentally. Additional specific chemical interactions between the surface and the ions are likely needed to predict this desorption correctly.

\section{ACKNOWLEDGMENTS}

We gratefully acknowledge F.A.M. Leermakers (Wageningen University) for helpful discussions.
[1] D. H. Napper, Polymeric Stabilisation of Colloidal Particles (Academic Press, London, 1983).

[2] R. H. Ottewill, J. Colloid Interface Sci. 58, 357 (1977).

[3] J. T. G. Overbeek, J. Colloid Interface Sci. 58, 408 (1977).

[4] J. M. H. M. Scheutjens, G. J. Fleer, M. A. Cohen Stuart, T. Cosgrove, and B. Vincent, Polymers at Interfaces (Chapman \& Hall, London, 1993).

[5] B. Mishra, B. B. Patel, and S. Tiwari, Nanomed.: Nanotechnol. Biol. Med. 6, 9 (2010).

[6] S. Farrokhpay, Adv. Colloid Interface Sci. 151, 24 (2009).

[7] C. Priest, A. Quinn, A. Postma, A. N. Zelikin, J. Ralston, and F. Caruso, Lab on a Chip 8, 2182 (2008).

[8] I. Wong and C.-M. Ho, Microfluid Nanofluid 7, 291 (2009).

[9] J. van der Gucht, E. Spruijt, M. Lemmers, and M. A. Cohen Stuart, J. Colloid Interface Sci. 361, 407 (2011).
[10] E. Spruijt, H. E. Bakker, J. Sprakel, M. A. Cohen Stuart, and J. van der Gucht, Soft Matter 7, 8281 (2011).

[11] P. G. De Gennes, J. Phys. France 37, 1445 (1976).

[12] P. G. De Gennes, Macromolecules 13, 1069 (1980).

[13] M. A. Cohen Stuart, G. J. Fleer, and B. H. Bijsterbosch, J. Colloid Interface Sci. 90, 310 (1982).

[14] M. A. Cohen Stuart, G. J. Fleer, and J. M. H. M. Scheutjens, J. Colloid Interface Sci. 97, 526 (1984).

[15] J. C. Dijt, M. A. Cohen Stuart, J. E. Hofman, and G. J. Fleer, Colloids and Surfaces 51, 141 (1990).

[16] G. P. van der Beek, M. A. Cohen Stuart, and T. Cosgrove, Langmuir 7, 327 (1991).

[17] J. Rubio and J. A. Kitchener, J. Colloid Interface Sci. 57, 132 (1976).

[18] E. Mubarekyan and M. M. Santore, Macromolecules 34, 7504 (2001). 
[19] B. Cattoz, W. M. de Vos, T. Cosgrove, M. Crossman, and S. W. Prescott, Langmuir 28, 6282 (2012).

[20] C. L. Cooper, T. Cosgrove, J. S. van Duijneveldt, M. Murray, and S. W. Prescott, Langmuir 29, 12670 (2013).

[21] G. P. van der Beek, M. A. Cohen Stuart, G. J. Fleer, and J. E. Hofman, Macromolecules 24, 6600 (1991).

[22] L. T. Zhuralev, Colloid Surface Physicochem. Eng. Aspect 173, 1 (2000).

[23] G. H. Bolt, J. Phys. Chem. 61, 1166 (1957).

[24] B. R. Postmus, F. A. M. Leermakers, and M. A. Cohen Stuart, Langmuir 24, 1930 (2008).

[25] W. M. de Vos, B. Cattoz, M. P. Avery, T. Cosgrove, and S. W. Prescott, Langmuir 30, 8425 (2014).

[26] S. K. Pattanayek and V. A. Juvekar, Macromolecules 35, 9574 (2002).

[27] P. M. Biesheuvel, W. M. de Vos, and V. M. Amoskov, Macromolecules 41, 6254 (2008).

[28] C. Marques and J.-F. Joanny, Macromolecules 23, 268 (1990).

[29] E. M. Blokhuis, K. I. Skau, and J. B. Avalos, J. Chem. Phys. 119, 3483 (2003).

[30] A. Johner, J.-F. Joanny, and M. Rubinstein, Europhys. Lett. 22, 591 (1993).

[31] J.-F. Joanny, Eur. Phys. J B: Cond. Matter Complex Systems 9 , 117 (1999).

[32] P. M. Biesheuvel, Eur. Phys. J. E: Soft Matter Biol. Phys. 16, 353 (2005).

[33] T. A. Vilgis, Phys. Rep. 336, 167 (2000).

[34] G. H. Fredrickson, The Equilibrium Theory of Inhomogeneous Polymers, International Series of Monographs on Physics No. 134 (Oxford Science Publications, Oxford, 2006).

[35] J. Bonet Avalos, J.-F. Joanny, A. Johner, and A. Semenov, Europhys. Lett. 35, 97 (1996).

[36] A. Semenov, J.-F. Joanny, and A. Johner, Theoretical and Mathematical models in Polymer Research, edited by A. Grosberg (Academic Press, Amsterdam, 1998).

[37] D. Andelman and J.-F. Joanny, Comptes Rendus de l'Académie des Sciences. Series IV - Physics 1, 1153 (2000).
[38] B. D. Storey and M. Z. Bazant, Phys. Rev. E 86, 056303 (2012).

[39] P. M. Biesheuvel and M. van Soestbergen, J. Colloid Interface Sci. 316, 490 (2007).

[40] D. McCormack, S. L. Carnie, and D. Y. C. Chan, J. Coll. Int. Sci. 169, 177 (1995).

[41] H. Gerischer, R. McIntyre, D. Scherson, and W. Storck, J. Phys. Chem. 91, 1930 (1987).

[42] H. Wang and L. Pilon, J. Power Sources 221, 252 (2013).

[43] W. B. S. de Lint, N. E. Benes, J. Lyklema, H. J. M. Bouwmeester, A. J. van der Linde, and M. Wessling, Langmuir 19, 5861 (2003).

[44] P. M. Biesheuvel, J. Phys: Cond. Matter 16, L499 (2004).

[45] I. Prigogine, P. Mazur, and R. Defay, J. de Chimie Physique et de Physicochimie Biologique 50, 146 (1953).

[46] M. J. Sparnaay, The Electrical Double Layer (Pergamon, Oxford, 1972).

[47] J. J. López-García, J. Horno, and C. Grosse, J. Colloid Interface Sci. 428, 308 (2014).

[48] I. Borukhov, D. Andelman, and H. Orland, Europhys. Lett. 32, 499 (1995).

[49] I. Borukhov, D. Andelman, and H. Orland, Macromolecules 31, 1665 (1998).

[50] W. M. de Vos, P. M. Biesheuvel, A. de Keizer, J. M. Kleijn, and M. A. Cohen Stuart, Langmuir 25, 9252 (2009).

[51] E. Spruijt and P. M. Biesheuvel, J. Phys.: Cond. Matter 26, 075101 (2014).

[52] N. A. M. Besseling and A. V. Korobko, Phys. Rev. Lett. 111, 186103 (2013)

[53] N. G. Hoogeveen, M. A. Cohen Stuart, and G. J. Fleer, J. Coll. Int. Sci. 182, 133 (1996).

[54] E. R. Nightingale Jr, J. Phys. Chem. 63, 1381 (1959).

[55] S. H. Behrens and D. G. Grier, J. Chem. Phys. 115, 6716 (2001).

[56] The $\mathrm{Si}_{-} \mathrm{SiO}_{2}$ system, edited by P. Balk (Elsevier, Amsterdam, 1988).

[57] D. Romeis, H. Merlitz, and J.-U. Sommer, J. Chem. Phys. 136, 044903 (2012). 